ESAIM: PROCEEDINGS, December 2012, Vol. 38, p. 319-334

F. Coquel, M. Gutnic, P. Helluy, F. Lagoutière, C. Rohde, N. Seguin, Editors

\title{
SHAPE OPTIMIZATION OF A SODIUM FAST REACTOR CORE
}

\author{
Emmanuel Dombre ${ }^{1}$, Grégoire Allaire $^{2}$, Olivier Pantz ${ }^{2}$ and Damien Schmitt ${ }^{3}$
}

\begin{abstract}
We apply in this paper a geometrical shape optimization method for the design of the core of a SFR (Sodium-cooled Fast Reactor) in order to minimize a thermal counter-reaction known as the sodium void effect. In this kind of reactors, by increasing the temperature, the core may become liable to a strong increase of reactivity, a key-parameter governing the chain-reaction at quasi-static states. We first use the one group energy diffusion model and give the generalization to the two groups energy equation. We then give some numerical results in the case of the one group energy equation. Note that the application of our method leads to some designs whose interfaces can be parametrized by very smooth curves which can stand very far from realistic designs. We don't explain here the method that it would be possible to use for recovering an operational design but there exists several penalization methods (see [2]) that could be employed to this end.
\end{abstract}

Résumé. On applique dans cet article une méthode d'optimisation géométrique dans le cadre de la conception d'un cœur de réacteur SFR (Sodium-cooled Fast Reactor, i.e. réacteur à neutron rapide refroidi au sodium) dans le but de minimiser une contre réaction thermique connue sous le nom d'effet de vidange sodium. Lorsqu'une augmentation de température survient, ce type de réacteur peut être sujet à une forte augmentation de réactivité, un paramètre clé dans le contrôle de la réaction en chaîne en régime quasi-statique. On a recours à l'équation de diffusion à un groupe puis on donne la généralisation du modèle d'optimisation pour l'équation de la diffusion à deux groupes d'énergie. On présente ensuite quelques résultats numériques obtenus dans le cas de l'équation à un groupe d'énergie. On note que l'application de cette méthode conduit à des designs de cœur présentant des interfaces très régulières qui sont loin d'un design de cœur faisable sur le plan technologique. A cet effet, de nombreuses méthodes de pénalisation (comme celles rencontrées dans 2]) pourraient être employées.

\section{INTRODUCTION}

We propose in this work to apply the geometric shape optimization method (see [6], 1]) in order to design a nuclear reactor core of generation IV which is cooled with sodium. The aim is to assess to what extent, such automatic way of improving the design could be positively employed. This kind of mathematical theory has already been investigated for optimizing the fuel reloading by the homogenization method (see [2]). We here restrict ourselves to the geometrical method. We have been working mainly on the one group diffusion equation, for which a whole presentation of the model is given in the first two sections of this paper. The sodium void effect that we want to reduce is mainly dependent on the geometry of the core and even the use of the simplified one group theory can lead to relevant results. However, this safety coefficient also has a component related to the energy spectrum. We give thus a extension of our model for this second state equation that is non

1 Edf R\&D, 1 avenue du Général De Gaulle 92141 Clamart

2 Centre de Mathématiques Appliquées, Ecole Polytechnique, 91128 Palaiseau

${ }^{3}$ Edf R\&D, département SINETICS, 1 avenue du Général De Gaulle 92141 Clamart

(C) EDP Sciences, SMAI 2012 
self-adjoint in the third section in order to pave the way for some future work. In section 4, we focus on some numerical results obtained with the one group theory. As the geometrical shape optimization method does not account for changes of topology, we need to use a Level-Set approach [4] in order to remesh the domain when some superposition of the interfaces occurs. Our algorithm is implemented thanks to the open source software FreeFem++ 7 that offers a suitable frame for this kind of problem.

\section{Statement of the Shape optimization Problem}

\subsection{Design of the core}

We assume that the reactor can be assimilated to a cylinder of radius $R$ and height $h$ and that the neutron flux $u$ is unchanged by rotation around the axis $r=0$. We thus have an axisymmetric geometry and we want to optimize the internal shape of the reactor that we define as the bounded set $\Omega=[0, R] \times[0, h]$.

In the frame of this work, we assume that the inside of the core is divided into four different parts $\Omega=\cup_{i=0}^{3} \omega_{i}$, each of them having some averaged physical properties. Three of them compose the active part of the core, responsible for the production of energy by fission reactions and playing an important role in terms of neutronic reactions. The last region is occupied by the reflector, an absorbing material mainly made of steel.

Each sub-domain $\omega_{i}$ is characterized by a diffusion coefficient $D^{i}$, and neutronic data, the macroscopic absorption and fission cross-sections (respectively denoted $\alpha^{i}$ and $\beta^{i}$ ). They depend only on the average density of sodium $d^{N a} \in \mathbb{R}^{+}$and, in the fissile region $\omega_{0}$, also on the concentration of plutonium $e$.

These parameters result from an accurate calculation according to the transport theory on a 33-groups energy mesh (with the code ERANOS 1 ) and homogenized in order to be used in the simplified modelings restricted to one or two groups to which we will from now on restrict our analysis. For more details about the way of calculating these parameters and their physical meaning, further information could be found in [8].

It follows, that for a given design and a given average density of sodium, the domain is characterized by

$$
\begin{aligned}
D\left(d^{N a}, \varphi, e\right) & :=D^{0}\left(d^{N a}, e\right) \varphi_{0}+\sum_{i=1}^{3} D^{i}\left(d^{N a}\right) \varphi_{i} \\
\alpha\left(d^{N a}, \varphi, e\right) & :=\quad \alpha^{0}\left(d^{N a}, e\right) \varphi_{0}+\sum_{i=1}^{3} \alpha^{i}\left(d^{N a}\right) \varphi_{i} ; \\
\beta\left(d^{N a}, \varphi, e\right) & :=\quad \beta^{0}\left(d^{N a}, e\right) \varphi_{0}+\sum_{i=1}^{3} \beta^{i}\left(d^{N a}\right) \varphi_{i} ;
\end{aligned}
$$

where $\varphi=\left(\varphi_{i}\right)_{i=0, \cdots, 3}=\left(\chi_{\omega_{i}}\right)_{i=0, \cdots, 3}=\chi_{\omega}$ stands for the family of characteristic functions of the $\omega_{i}$.

\subsection{State equation - The one group energy diffusion equation}

The behavior of the core is in a first approximation described by the eigenvector $u$ of smallest eigenvalue $\lambda$ of the one group energy diffusion equation

$$
\begin{cases}-r^{-1} \operatorname{div}(r D \nabla u)+\alpha u=\lambda \gamma u, & \text { in } \cup_{i} \omega_{i}, \\ {[u]=0} & \text { on } \Gamma, \\ {\left[D \frac{\partial u}{\partial n}\right]=0} & \text { on } \Gamma, \\ u=0 & \text { on } \Gamma_{D},\end{cases}
$$

where $\Gamma_{D}$ is the part of boundary of the domain $\Omega$ other than the symmetry axis $r=0$,

$$
\Gamma_{D}:=\partial \Omega \backslash\{(r, z) \text { such that } r=0\},
$$

\footnotetext{
${ }^{1}$ Used for core calculations in neutrons fast reactors
} 
whereas $\Gamma=\Omega \backslash \bigcup_{i=0}^{4} \omega_{i}$ is the interface between the sub-domains and $\gamma:=\nu \beta, \nu$ being the average number of neutrons produced by fission. This equation results from the equilibrium between each potential reactions happening into the core: fission, absorption and leakage.

Weak formulation. The weak formulation of (4) consists in finding $(\lambda, u) \in \mathbb{R} \times V$, where

$$
V:=\left\{u \in L^{2}(\Omega) \text { such that } \sqrt{r} \nabla u \in L^{2}(\Omega) \text { and } u=0 \text { on } \Gamma_{D}\right\},
$$

such that for every $v \in V$,

$$
a_{d^{N a}, \varphi, e}(u, v)=\lambda b_{d^{N a}, \varphi, e}(u, v),
$$

where

$$
a_{d^{N a}, \varphi, e}(u, v)=\int_{\Omega}(D \nabla u \cdot \nabla v+\alpha u v) d V
$$

and

$$
b_{d^{N a}, \varphi, e}(u, v)=\int_{\Omega} \gamma u v d V
$$

where $d V:=r d r d z$. We denote by $u\left(d^{N a}, \varphi, e\right)$ and $\lambda\left(d^{N a}, \varphi, e\right)$ the solution of this equation.

\subsection{Setting of the Optimization Problem}

In this section, we introduce the design problem we aim to solve. Our main objective consists in minimizing the sodium void effect, that is the variation of the reactivity, which is equal to $\rho\left(d^{N a}, \varphi, e\right):=1-\lambda\left(d^{N a}, \varphi, e\right)$. The lower is the first eigenvalue, the bigger is the reactivity. In general, the decrease of the average sodium density leads to an increase of the reactivity. The variation is strongly related to the geometry of the core. To measure the sodium void effect, we introduce the cost function

$$
J_{1}(\varphi, e):=\lambda_{1}(\varphi, e)-\lambda_{2}(\varphi, e),
$$

where $\lambda_{1}$ and $\lambda_{2}$ are the first eigenvalues of 5 for a concentration of sodium $d_{1}^{N a}$ in the nominal state and $d_{2}^{N a}$ in a perturbed one, with $d_{2}^{N a}=\left(1-10^{-2}\right) d_{1}^{N a}$. We set

$$
\lambda_{k}(\varphi, e):=\lambda\left(d_{k}^{N a}, \varphi, e\right), \quad k=1,2 .
$$

and in a similar fashion define

$$
\left.u_{k}(\varphi, e):=u\left(d_{k}^{N a}, \varphi, e\right)\right), \quad k=1,2 .
$$

As one can expect, minimizing only the sodium void effect will not lead to a realistic design and other performance or safety requirements should be taken into account. First, we would like the reactivity in the nominal state to be close to a target value, or equivalently, $\lambda_{1}$ to be close to a given constant $\lambda_{0}$. To this end, we introduce a second cost function

$$
J_{2}(\varphi, e):=\left(\lambda_{1}(\varphi, e)-\lambda_{0}\right)^{2} .
$$

Another safety requirement stems from the power distribution which must be as uniform as possible in the core, and more particularly in the region of fissile material $\omega_{0}$. We usually define the shape factor as the ratio between the maximum value of the rate of fission $\tau_{1}(\varphi, e):=\beta^{0}\left(d_{1}^{N a}, e\right) u_{1}(\varphi, e)$ in a nominal state and its mean value on the fissile region $-u_{1}$ is the neutron flux in the nominal state, that is for a sodium concentration equal to $d_{1}^{N a}$. As the infinity norm is not differentiable, we replace the classical shape factor by the following approximation (for a large enough value of $s$ ) :

$$
\begin{gathered}
J_{3}(\varphi, e):=\operatorname{Vol}\left(\varphi_{0}\right)^{\frac{s-1}{s}}\left(\int_{\Omega} \tau_{1} d V_{0}\right)^{-1}\left|\int_{\Omega} \tau_{1}^{s} d V_{0}\right|^{1 / s}, \\
\operatorname{Vol}(\varphi):=\int_{\Omega} \varphi d V
\end{gathered}
$$


where $d V_{0}:=\varphi_{0} d V$ is the integration over the fissile region. Finally, we have to take into account an additional constraint for the volume $\operatorname{Vol}\left(\omega_{0}\right)$ of the fissile region $\omega_{0}$. Its volume must be kept constant during the simulation in order to obtain designs that have approximately the same power. Thus our aim is to minimize a weighted sum of the cost $J_{k}, k=1, \cdots, 3$ over the set

$$
\begin{array}{r}
\mathcal{U}_{a d}:=\left\{(\omega, e) \in\left(\mathcal{V}^{4}, L^{\infty}(\Omega)\right) \text { such that } \forall i, j=0, \cdots, 3, \quad \omega_{i} \subset \Omega, \quad \omega_{i} \cap \omega_{j}=\emptyset, \quad \bar{\Omega}=\bigcup_{k=0}^{3} \overline{\omega_{i}}\right. \\
\left.e(x) \in\left[e_{\text {min }}, e_{\text {max }}\right] \text { for all } x \in \omega_{0} \text { and } \operatorname{Vol}\left(\omega_{0}\right)=V_{0}\right\}
\end{array}
$$

where $V_{0}$ is a positive real lower than the total volume $\operatorname{Vol}(\Omega)$ whereas $e_{\min }$ and $e_{\max }$ are respectively the minimal and maximal density of plutonium in $\omega_{0}$. The minimization problem can be stated as

$$
\underset{(\omega, e) \in \mathcal{U}_{a d}}{\arg \min }\{J(\omega, e)\}
$$

where

$$
J(\varphi, e):=s_{1} J_{1}(\varphi, e)+s_{2} J_{2}(\varphi, e)+s_{3} J_{3}(\varphi, e)
$$

and $\left(s_{k}\right)_{k=1,2,3}$ are given positive weights.

Remark 1. In order to simplify the notations, we will sometimes - as in the definition of the problem (12) or the definition of $\left.\mathcal{U}_{a d} \sqrt{11}\right)$ - write $F(\omega)$ instead of $F\left(\chi_{\omega}\right)$ when $F$ is a map defined on $\left(L^{\infty}(D)\right)^{r}$ and $\omega \in \mathcal{V}^{r}$ is a family of open subsets of $\Omega\left(r \in \mathbb{N}^{*}\right)$.

To minimize $J$, we alternatively perform a parametric optimization with respect to the concentration of plutonium $e$ and a geometric optimization with respect to the shape $\omega$ based in both cases on a gradient method.

We first differentiate in this section $J$ with respect to the variable $e$. This can be achieved by using the adjoint-method which allows us to obtain a simple expression of the gradient of $J$ for any increment $\delta e$ of the plutonium concentration at the price of one additional calculation of an adjoint state $p_{1}(\omega, e)$ given by

$$
\begin{cases}-r^{-1} \operatorname{div}\left(r D_{1} \nabla p_{1}\right)+\alpha_{1} p_{1}-\lambda_{1} \gamma_{1} p_{1}=\ell_{\omega, e} & \text { in } \cup_{i} \omega_{i} \\ {\left[p_{1}\right]=0} & \text { on } \Gamma \\ {\left[\frac{\partial p_{1}}{\partial n}\right]=0} & \text { on } \Gamma \\ p_{1}=0 & \text { on } \Gamma_{D}\end{cases}
$$

where

$$
\ell_{\omega, e}=V\left(\omega_{0}\right)^{\frac{s-1}{s}}\left(\int_{\omega_{0}} \beta_{1}^{0} u_{1} d V\right)^{-2}\left(\int_{\omega_{0}}\left(\beta_{1}^{0} u_{1}\right)^{s} d V\right)^{\frac{1-s}{s}}\left[\left(\int_{\omega_{0}}\left(\beta_{1}^{0} u_{1}\right)^{s} d V\right) \beta_{1}^{0}-\left(\int_{\omega_{0}} \beta_{1}^{0} u_{1} d V\right)\left(\beta_{1}^{0}\right)^{s} u_{1}^{s-1}\right]
$$

on $\omega_{0}, \ell_{\omega, e}=0$ on $\Omega \backslash \omega_{0}$ and

$$
D_{k}(\varphi, e)=D\left(d_{k}^{N a}, \varphi, e\right) ; \quad \alpha_{k}(\varphi, e)=\alpha\left(d_{k}^{N a}, \varphi, e\right) ; \quad \beta_{k}(\varphi, e)=\beta\left(d_{k}^{N a}, \varphi, e\right), \quad k=1,2
$$


Proposition 1.1. The parametric derivative $\partial J / \partial e$ in a direction $\delta e \in L^{\infty}\left(\omega_{0}\right)$ is given by the following equation

$$
\begin{aligned}
\left\langle\frac{\partial J}{\partial e}, \delta e\right\rangle= & V\left(\omega_{0}\right)^{\frac{s-1}{s}}\left(\int_{\omega_{0}} \beta_{1}^{0} u_{1} d V\right)^{-2}\left|\int_{\omega_{0}}\left(\beta_{1}^{0} u_{1}\right)^{s} d V\right|^{\frac{1-s}{s}} \\
& \left(\int_{\omega_{0}} \beta_{1}^{0} u_{1} r d r d z \int_{\omega_{0}}\left(\beta_{1}^{0}\right)^{s-1} \frac{d \beta_{1}^{0}}{d e} u_{1}^{s} \delta e d V-\int_{\omega_{0}}\left(\beta_{1}^{0} u_{1}\right)^{s} r d r d z \int_{\omega_{0}} \frac{d \beta_{1}^{0}}{d e} u_{1} \delta e d V\right) \\
& +\sum_{k=1,2}\left[\int_{\omega_{0}}\left(\frac{d D_{k}}{d e} \nabla u_{k} \cdot \nabla p_{k}+\frac{d \alpha_{k}}{d e} u_{k} p_{k}-\lambda_{k} \frac{d \gamma_{k}}{d e} u_{k} p_{k}\right) \delta e d V\right]
\end{aligned}
$$

where $p_{1}$ is the solution of the adjoint-state equation (13), $p_{2}=-u_{2}$ and $u_{k}$ the solution of the 1-group equation (8) in the state $k$.

\subsection{Adjoint equation}

In order to prove Proposition 1.1, we introduce a Lagrangian $\mathcal{L}$ as the sum of the cost function $J$ (written for independent variables) and the constraint provided by the state equation.

$$
\mathcal{L}\left(\varphi, e, \hat{u}_{1}, \hat{u}_{2}, \hat{p}_{1}, \hat{p}_{2}, \hat{\lambda}_{1}, \hat{\lambda}_{2}\right)=j\left(\varphi, e, \hat{u}_{1}, \hat{u}_{2}, \hat{\lambda}_{1}, \hat{\lambda}_{2}\right)+\sum_{k=1,2} a_{\varphi, e}^{k}\left(\hat{u}_{k}, \hat{p}_{k}\right)-\hat{\lambda}_{k} b_{\varphi, e}^{k}\left(\hat{u}_{k}, \hat{p}_{k}\right)
$$

where

$$
j\left(\varphi, e, \hat{u}_{1}, \hat{u}_{2}, \hat{\lambda}_{1}, \hat{\lambda}_{2}\right):=\hat{\lambda}_{1}-\hat{\lambda}_{2}+\left(\hat{\lambda}_{1}-\lambda_{0}\right)^{2}+V\left(\varphi_{0}\right)^{\frac{s-1}{s}}\left(\int_{\Omega} \beta_{1}^{0} \hat{u}_{1} d V_{0}\right)^{-1}\left|\int_{\Omega}\left(\beta_{1}^{0} \hat{u}_{1}\right)^{s} d V_{0}\right|^{1 / s}
$$

while

$$
a_{\varphi, e}^{k}:=a_{d_{k}^{N a}, \varphi, e} \text { and } b_{\varphi, e}^{k}:=b_{d_{k}^{N a}, \varphi, e} .
$$

Remark 2. The dependence of $a_{\varphi, e}^{k}$ and $b_{\varphi, e}^{k}$ with respect to the partition $\varphi$ and the concentration in plutonium $e$ will sometimes be understood, and we will use the notations $a^{k}$ and $b^{k}$ instead.

We recall that $D_{k}, \alpha_{k}$ and $\gamma_{k}$ are the values of $D, \alpha$ and $\gamma$ for the concentration of sodium $d_{k}^{N a}$ for a given partition $\varphi$ of the domain $\Omega$ and for a sodium concentration $e$.

For all $\left(\hat{p}_{1}, \hat{p}_{2}\right) \in V^{2}$, we have

$$
J(\varphi, e)=\mathcal{L}\left(\varphi, e, u_{1}(\varphi, e), \lambda_{1}(\varphi, e), u_{2}(\varphi, e), \lambda_{2}(\varphi, e), \hat{p}_{1}, \hat{p}_{2}\right)
$$

which yields by composed derivation to

$$
\left\langle\frac{\partial J}{\partial e}, \delta e\right\rangle=\left\langle\frac{\partial \mathcal{L}}{\partial e}, \delta e\right\rangle+\sum_{k=1,2}\left\langle\frac{\partial \mathcal{L}}{\partial \hat{u}_{k}},\left\langle\frac{\partial u_{k}}{\partial e}, \delta e\right\rangle\right\rangle+\frac{\partial \mathcal{L}}{\partial \hat{\lambda}_{k}}\left\langle\frac{\partial \lambda_{k}}{\partial e}, \delta e\right\rangle .
$$

Let us remark that it is well known that both $u_{k}$ and $\lambda_{k}$ are differentiable with respect to $e$ as maps with values in $V$ and $\mathbb{R}$ respectively. In particular, the application of the chain rule to obtain (16) is licit.

The adjoint-states $p_{k}$ are then defined in such a way that they make the derivatives of $\mathcal{L}$ with respect to the state variables $\left(\hat{u}_{k}, \hat{\lambda}_{k}\right)$ vanish. As

$$
\left\langle\frac{\partial u_{k}}{\partial e}, \delta e\right\rangle \in V_{k}:=\left\{q \in V \text { such that } b^{k}\left(u_{k}, q\right)=0\right\}
$$


this leads to the following weak formulation for the state $k$

$$
\left\{\begin{array} { l } 
{ \langle \frac { \partial \mathcal { L } } { \partial \hat { u } _ { k } } ( \omega , e , u _ { k } , \lambda _ { k } , p _ { k } ) , q \rangle = 0 \forall q \in V _ { k } } \\
{ \frac { \partial \mathcal { L } } { \partial \hat { \lambda } _ { k } } ( \omega , e , u _ { k } , \lambda _ { k } , p _ { k } ) = 0 }
\end{array} \Longleftrightarrow \left\{\begin{array}{l}
a^{k}\left(q, p_{k}\right)-\lambda_{k} b^{k}\left(q, p_{k}\right)=-\left\langle\frac{\partial j}{\partial \hat{u}_{k}}, q\right\rangle \forall q \in V_{k} \\
b^{k}\left(u_{k}, p_{k}\right)=\frac{\partial j}{\partial \hat{\lambda}_{k}}
\end{array}\right.\right.
$$

It is easily seen that the solution of the adjoint equation for $k=2$ is simply $p_{2}(\varphi, e):=-u_{2}(\varphi, e)$. On the other hand, we get that $p_{1}(\varphi, e) \in V$ is such that for all $q_{1} \in V_{1}$,

$$
\begin{aligned}
a_{\varphi, e}^{k}\left(p_{1}, q_{1}\right)-\lambda_{1} b_{\varphi, e}^{1}\left(p_{1}, q_{1}\right) & =\ell_{\varphi, e}\left(q_{1}\right) \\
b_{\varphi, e}^{1}\left(p_{1}, u_{1}\right) & =1+2\left(\lambda_{1}-\lambda_{0}\right)
\end{aligned}
$$

where

$$
\begin{aligned}
\ell_{\varphi, e}\left(q_{1}\right):=V\left(\varphi_{0}\right)^{\frac{s-1}{s}}\left(\int_{\Omega} \beta_{1}^{0} u_{1} d V_{0}\right)^{-2}\left(\int_{\Omega}\left(\beta_{1}^{0} u_{1}\right)^{s} d V_{0}\right)^{\frac{1-s}{s}} & {\left[\left(\int_{\Omega}\left(\beta_{1}^{0} u_{1}\right)^{s} d V_{0}\right) \int_{\Omega} \beta_{1}^{0} q_{1} d V_{0}-\left(\int_{\Omega} \beta_{1}^{0} u_{1} d V_{0}\right) \int_{\Omega}\left(\beta_{1}^{0}\right)^{s} u_{1}^{s-1} q_{1} d V_{0}\right] . }
\end{aligned}
$$

It can be easily proved that 18,19 admits a unique solution, which will be denoted by $p_{1}(\varphi, e)$. Indeed, let $\bar{p}_{1}=p_{1}-\left(1+2\left(\lambda_{1}-\lambda_{0}\right)\right) u_{1}$. Then $p_{1}$ is a solution if and only if $\bar{p}_{1} \in V_{1}$ and for all $\bar{q}_{1} \in V_{1}$ we have

$$
a^{1}\left(\bar{p}_{1}, \bar{q}_{1}\right)-\lambda_{1} b^{1}\left(\bar{p}_{1}, \bar{q}_{1}\right)=\ell\left(\bar{q}_{1}\right)
$$

Moreover, it can be checked that the bilinear form $a^{1}-\lambda_{1} b^{1}$ is coercive on $V_{1}$ and the conclusion follows from the application of the Lax-Milgram Theorem.

It remains to evaluate the sensitivity of $\mathcal{L}$ with respect to the design variable $e$. Some calculations provide for any $\delta e$

$$
\begin{aligned}
\left\langle\frac{\partial \mathcal{L}}{\partial e}, \delta e\right\rangle=V\left(\omega_{0}\right)^{\frac{s-1}{s}} & \left(\int_{\omega_{0}} \beta_{1}^{0} \hat{u}_{1} d V\right)^{-2}\left|\int_{\omega_{0}}\left(\beta_{1}^{0} \hat{u}_{1}\right)^{s} d V\right|^{\frac{1-s}{s}} \\
& \left(\int_{\omega_{0}} \beta_{1}^{0} \hat{u}_{1} d V \int_{\omega_{0}}\left(\beta_{1}^{0}\right)^{s-1} \frac{d \beta_{1}^{0}}{d e} \hat{u}_{1}^{s} \delta e d V-\int_{\omega_{0}}\left(\beta_{1}^{0} \hat{u}_{1}\right)^{s} d V \int_{\omega_{0}} \frac{d \beta_{1}^{0}}{d e} \hat{u}_{1} \delta e d V\right) \\
& +\sum_{k=1,2}\left[\int_{\Omega}\left(\frac{d D_{k}}{d e} \nabla \hat{u}_{k} \cdot \nabla \hat{p}_{k}+\frac{d \alpha_{k}}{d e} \hat{u}_{k} \hat{p}_{k}-\hat{\lambda}_{k} \frac{d \gamma_{k}}{d e} \hat{u}_{k} \hat{p}_{k}\right) \delta e d V\right]
\end{aligned}
$$

By applying (16) at $\left(u_{k}, p_{k}, \lambda_{k}\right)_{k=1,2}$, we get the announced expression 14 of the parametric gradient of $J$ with respect to the concentration of sodium.

\subsection{Gradient method}

In order to minimize our cost function $J$ with respect to $e$, we apply a projected gradient algorithm. We construct a minimizing sequence $e_{n \in \mathbb{N}}$ recursively, setting

$$
e_{n+1}=\max \left(\min \left(e_{n}-\mu \delta e_{n}, e_{\max }\right), e_{\min }\right)
$$


where $\delta e_{n} \in L^{2}\left(\omega_{0}\right)$ is such that for all $\delta e \in L^{2}\left(\omega_{0}\right)$,

$$
\left(\delta e_{n}, \delta e\right)_{L^{2}}=\left\langle\frac{\partial J}{\partial e}\left(\omega, e_{n}\right), \delta e\right\rangle
$$

and $\mu>0$ is a small time step, starting with an initial uniform design parameter $e_{0}$. Note that $\delta e_{n}$ is correctly defined provided the solutions of the state and adjoint equations are regular enough. Moreover, from the practical point of view, it could be of some interest to regularize a little bit the solution $\delta e_{n}$ of $(20)$ by replacing the $L^{2}$ scalar product by $\left(\delta e_{n}, \delta e\right)_{L^{2}}+\varepsilon\left(\nabla \delta e_{n}, \nabla \delta e\right)_{L^{2}}$. However, it should be reminded that with such a regularization, fix points of the algorithm are not minimizers of the problem, because the operator $\left.\max \left(\min \left(\cdot, e_{\max }\right), e_{\min }\right)\right)$ is not the orthogonal projection onto $\left[e_{\min }, e_{\max }\right]$ for such a choice of scalar product. Nevertheless, if $\varepsilon$ is chosen small enough, it has little impact on the solution obtained. A more rigorous approach would have been to add Lagrange multipliers to take into account those constraints and to use an Uzawa algorithm, what is, unfortunately, a lot more time consuming.

\section{Geometric Shape optimization}

Let us now consider the minimization of $J$ with respect to the shape $\omega$. To this end, we perform small perturbations of the sub domains $\omega_{i}$ by convecting them along a vector field $\theta$ of the domain $\Omega$. More precisely, let $\theta \in W^{1, \infty}\left(\Omega ; \mathbb{R}^{3}\right)$ such that $\theta \cdot n=0$ on $\partial \Omega$, we set $\omega_{i}(t)=X_{\theta}\left(t, \omega_{i}\right)$ where $X_{\theta}$ is the map from $\Omega \times \mathbb{R}$ into $\Omega$ defined by

$$
\left\{\begin{array}{l}
\dot{X}_{\theta}(x, t)=\theta\left(X_{\theta}(x, t)\right) \quad \text { for all }(t, x) \in \mathbb{R} \times \Omega \\
X_{\theta}(x, 0)=x
\end{array}\right.
$$

In the following, if no confusion is possible, we will use the notation $X$ instead of $X_{\theta}$. The shape derivative at $\omega$ of a functional $J(\omega, e)$ is given by the derivative at $t=0$ of the map $t \mapsto J(\omega(t), e)$.

\subsection{Computation of the shape derivative}

In this section, we compute the derivative of the cost function $J$ with respect to the shape of the partition $\omega$ of $\Omega$.

Proposition 2.1. The shape derivative of our cost function in any direction $\theta$ is given by :

$$
\begin{aligned}
\left\langle\frac{\partial J}{\partial \omega}, \theta\right\rangle & =\operatorname{Vol}\left(\omega_{0}\right)^{\frac{s-1}{s}}\left(\int_{\omega_{0}} \beta_{1} u_{1} d V\right)^{-1}\left|\int_{\omega_{0}}\left(\beta_{1} u_{1}\right)^{s} d V\right|^{1 / s}\left[\frac{s-1}{s} \operatorname{Vol}\left(\omega_{0}\right)^{-1} \int_{\partial \omega_{0}}(\theta \cdot n) r d s\right. \\
& \left.-\left(\int_{\omega_{0}} \beta_{1} u_{1} d V\right)^{-1} \int_{\partial \omega_{0}} \beta_{1}^{0} u_{1}(\theta \cdot n) r d s+\frac{1}{s}\left(\int_{\omega_{0}}\left(\beta_{1} u_{1}\right)^{s} d V\right)^{-1} \int_{\partial \omega_{0}}\left(\beta_{1}^{0} u_{1}\right)^{s}(\theta \cdot n) r d s\right] \\
& -\sum_{k=1,2} \int_{\Gamma}\left(\left[D_{k}\right] \frac{\partial u_{k}}{\partial \tau} \frac{\partial p_{k}}{\partial \tau}-\left[D_{k}^{-1}\right]\left(D_{k} \frac{\partial u_{k}}{\partial n}\right)\left(D_{k} \frac{\partial p_{k}}{\partial n}\right)+\left[\alpha_{k}\right] u_{k} p_{k}-\lambda_{k}\left[\gamma_{k}\right] u_{k} p_{k}\right)(\theta \cdot n) d V
\end{aligned}
$$

where $\beta_{1}^{0}=\beta^{0}\left(d_{1}^{N a}, e\right)$.

The fast derivation method of Céa we have used for the computation of the differential with respect to the concentration of plutonium $e$ can not be applied directly, because the Eulerian differential of the state does not belong to $V$ (in particular, the chain rule used to derive (16) can not be applied).

This problem can be circumvented by different approaches. The most classical one consists in performing a change of variables in order to rewrite the state equation on fix sub-domains rather than $\omega(t)$, another one to use a different definition for the Lagrangian taking explicitly into account the equations of transition on the interfaces between the sub-domains. In this article, we propose to follow another path as in 5. Roughly 
speaking it consists first to apply the fast derivation method by assuming the coefficients $D, \beta$ and $\alpha$ to be regular, then to "pass to the limit" into the expression of the derivative obtained.

To this end, we generalize the notion of shape derivation to cases where the map $\varphi$ is not necessarily a family of characteristic functions. Let $\varphi$ be a partition of unity of the domain $\Omega$, we define $\varphi(t)$ as the partition defined by

$$
\varphi_{i}(t)(x)=\varphi_{i}(X(x,-t)) \text { for } i=0, \cdots, 3,
$$

and we define the shape derivative of the functional $J(\varphi, e)$ as the derivative at time $t=0$ of the map $t \mapsto$ $J(\varphi(t), e)$. Note that both definition coincide if $\varphi$ is a family of characteristic functions. Indeed, if $\varphi=\chi_{\omega}$, we have $J(\varphi(t), e)=J\left(\chi_{\omega(t)}, e\right)=J(\omega(t), e)$.

The computation of the classical derivative is then obtained in a two steps process. We first compute the shape derivative for regular partitions $\varphi$ and extend (formally) this expression when $\varphi$ is a family of characteristic functions by passing to the limit.

\subsubsection{Regular case}

If $\varphi$ is a regular partition of the unity, the fast derivation method of Céa can be applied. Using the same Lagrangian than the one used to derive the derivative of $J$ with respect to $e$ and the same adjoint state defined by 18,19, , we get that

$$
\left\langle\frac{\partial J}{\partial \omega}(\varphi, e), \theta\right\rangle=\left\langle\frac{\partial \mathcal{L}}{\partial \omega}\left(\varphi, e, u_{1}, u_{2}, p_{1}, p_{2}\right), \theta\right\rangle
$$

We just have to compute the shape derivative of $\mathcal{L}$ to conclude. First, as

$$
\operatorname{Vol}\left(\varphi_{0}(t)\right)=\int_{\Omega} \varphi_{0}(t) d V=\int_{\Omega} \varphi_{0}(X(x,-t)) d V,
$$

and $\dot{X}(x, t=0)=\theta$, we get

$$
\left\langle\frac{\partial \mathrm{Vol}}{\partial \omega}, \theta\right\rangle=-\int_{\Omega} \nabla \varphi_{0} \cdot \theta d V
$$

A similar computation (even if more tedious), leads to

$$
\begin{aligned}
\left\langle\frac{\partial j}{\partial \omega}, \theta\right\rangle= & \operatorname{Vol}\left(\varphi_{0}\right)^{\frac{s-1}{s}}\left(\int_{\Omega} \beta_{1}^{0} \hat{u}_{1} d V_{0}\right)^{-1}\left(\int_{\Omega}\left(\beta_{1}^{0} \hat{u}_{1}\right)^{s} d V_{0}\right)^{1 / s}\left[\frac{1-s}{s} \operatorname{Vol}\left(\varphi_{0}\right)^{-1} \int_{\Omega}\left(\nabla \varphi_{0} \cdot \theta\right) d V\right. \\
& \left.+\left(\int_{\Omega} \beta_{1}^{0} \hat{u}_{1} d V_{0}\right)^{-1} \int_{\Omega} \beta_{1}^{0} \hat{u}_{1}\left(\nabla \varphi_{0} \cdot \theta\right) d V-\frac{1}{s}\left(\int_{\Omega}\left(\beta_{1}^{0} \hat{u}_{1}\right)^{s} d V_{0}\right)^{-1} \int_{\Omega}\left(\beta_{1}^{0} \hat{u}_{1}\right)^{s}\left(\nabla \varphi_{0} \cdot \theta\right) d V\right]
\end{aligned}
$$

The computation of the differential of the last part of the Laplacian turns out to reduce itself to the computation of the variation of the coefficients $D_{k}, \alpha_{k}$ and $\beta_{k}$. As

$$
D_{k}(\varphi(t), e)(x)=\sum_{i} D_{k}^{i}(e) \varphi_{i}(X(-t, x))
$$

we get deriving this expression with respect to $t$ at time $t=0$

$$
\left\langle\frac{\partial D_{k}}{\partial \omega}, \theta\right\rangle=-\sum_{i} D_{k}^{i}(e) \nabla \varphi_{i} \cdot \theta
$$


where $D_{k}^{i}(e):=D^{i}\left(d_{k}^{N a}, e\right)$. The same expression could be derived for $\alpha_{k}$ and $\beta_{k}$. From 22, 23, and (24), we obtain that

$$
\begin{aligned}
\left\langle\frac{\partial J}{\partial \omega}, \theta\right\rangle & =\operatorname{Vol}\left(\varphi_{0}\right)^{\frac{s-1}{s}}\left(\int_{\Omega} \beta_{1}^{0} u_{1} d V_{0}\right)^{-1}\left|\int_{\Omega}\left(\beta_{1}^{0} u_{1}\right)^{s} d V_{0}\right|^{1 / s}\left[\frac{1-s}{s} \operatorname{Vol}\left(\varphi_{0}\right)^{-1} \int_{\Omega}\left(\nabla \varphi_{0} \cdot \theta\right) d V\right. \\
+ & \left.\left(\int_{\Omega} \beta_{1}^{0} u_{1} d V_{0}\right)^{-1} \int_{\Omega} \beta_{1}^{0} u_{1}\left(\nabla \varphi_{0} \cdot \theta\right) d V-\frac{1}{s}\left(\int_{\Omega}\left(\beta_{1}^{0} u_{1}\right)^{s} d V_{0}\right)^{-1} \int_{\Omega}\left(\beta_{1}^{0} u_{1}\right)^{s}\left(\nabla \varphi_{0} \cdot \theta\right) d V\right] \\
& -\sum_{\substack{k=1,2 \\
i=0, \cdots, 3}} \int_{\Omega}\left(D_{k}^{i} \nabla u_{k} \cdot \nabla p_{k}+\alpha_{k}^{i} u_{k} p_{k}-\lambda_{k} \gamma_{k}^{i} u_{k} p_{k}\right)\left(\nabla \varphi_{i} \cdot \theta\right) d V
\end{aligned}
$$

\subsubsection{Passage to the limit}

Let $\omega$ be a subdivision of the domain $\Omega$. Up to a small regularization, there exists a sequence $\varphi^{\varepsilon}$ of partition of the unity such that $\varphi^{\varepsilon}$ converges toward $\varphi_{\omega}$ into the space functions of bounded variation (it can for instance be obtained by mollification). In order to obtain the shape differential of $J(\omega, e)$ we formally pass to the limit in 25) on the differential of $J\left(\varphi^{\varepsilon}, e\right)$.

Let $F^{\varepsilon}$ be a sequence of continuous function on $\Omega$ converging toward $F$, for all $i=0, \cdots, 3$, we have

$$
\int_{\Omega} F^{\varepsilon}\left(\nabla \varphi_{i}^{\varepsilon} \cdot \theta\right) d V \stackrel{\varepsilon \rightarrow 0}{\longrightarrow}-\int_{\partial \omega_{i}} F(\theta \cdot n) r d s
$$

where $n$ is the outward normal to $\omega_{i}$. Unfortunately, it is not reasonable to pass directly to the limit in the expression of $\partial J / \partial \omega\left(\varphi^{\varepsilon}, e\right)$ using (26). A special treatment is needed for the convergence of the term

$$
s_{k}^{\varepsilon}:=\sum_{i=0, \cdots 3} \int_{\Omega} D_{k}^{i} \nabla u_{k}^{\varepsilon} \cdot \nabla p_{k}^{\varepsilon}\left(\nabla \varphi_{i}^{\varepsilon} \cdot \theta\right) d V .
$$

Indeed, neither $\nabla u_{k}$ nor $\nabla p_{k}$ can be expected to be continuous on $\Omega$. Nevertheless, we can assume both $\partial u_{k}^{\varepsilon} / \partial \tau$ and $D_{k}^{\varepsilon} \partial u_{k}^{\varepsilon} / \partial n$ to be convergent (where $n$ is an extension of the normal to the interface $\Gamma$ between the subdomains and $\tau$ is the tangent vector to the interface and $D_{k}^{\varepsilon}:=D_{k}\left(\varphi^{\varepsilon}, e\right)$.). The term $s_{k}^{\varepsilon}$ could be split in two parts,

$$
s_{k, n}^{\varepsilon}:=\sum_{i=0, \cdots 3} \int_{\Omega} D_{k}^{i} \frac{\partial u_{k}^{\varepsilon}}{\partial n} \frac{\partial p_{k}^{\varepsilon}}{\partial n}\left(\nabla \varphi_{i}^{\varepsilon} \cdot \theta\right) d V
$$

and

$$
s_{k, \tau}^{\varepsilon}:=\sum_{i=0, \cdots 3} \int_{\Omega} D_{k}^{i} \frac{\partial u_{k}^{\varepsilon}}{\partial \tau} \frac{\partial p_{k}^{\varepsilon}}{\partial \tau}\left(\nabla \varphi_{i}^{\varepsilon} \cdot \theta\right) d V .
$$

We can directly use 26 to compute the limit of $s_{k, \tau}^{\varepsilon}$ and we get

$$
s_{k, \tau}^{\varepsilon} \rightarrow-\int_{\Gamma}\left[D_{k}\right] \frac{\partial u_{k}^{\varepsilon}}{\partial \tau} \frac{\partial p_{k}^{\varepsilon}}{\partial \tau}(\theta \cdot n) r d s
$$

In order to compute the limit of the first term $s_{k, n}^{\varepsilon}$ we rewrite it as

$$
s_{k, n}^{\varepsilon}=-\int_{\Omega}\left(D_{k}^{\varepsilon} \frac{\partial u_{k}^{\varepsilon}}{\partial n}\right)\left(D_{k}^{\varepsilon} \frac{\partial p_{k}^{\varepsilon}}{\partial n}\right)\left(\nabla\left(\left(D_{k}^{\varepsilon}\right)^{-1}\right) \cdot \theta+\left(D_{k}^{\varepsilon}\right)^{-2} \sum_{i}\left(\nabla D_{k}^{i} \cdot \theta\right) \varphi_{i}^{\varepsilon}\right) d V
$$


As $\left(D_{k}^{\varepsilon}\right)^{-1}$ converges toward $D_{k}^{-1}$ in the space of functions of bounded variation, we can pass to the limit in this expression to obtain that

$$
\begin{aligned}
s_{k, n}^{\varepsilon} \rightarrow \int_{\Gamma}\left(D_{k} \frac{\partial u_{k}}{\partial n}\right)\left(D_{k} \frac{\partial p_{k}}{\partial n}\right)\left[D_{k}^{-1}\right](\theta \cdot n) r d s & \\
& -\sum_{i=0, \cdots, 3} \int_{\omega_{i}}\left(D_{k} \frac{\partial u_{k}}{\partial n}\right)\left(D_{k} \frac{\partial p_{k}}{\partial n}\right)\left(\nabla\left(\left(D_{k}^{i}\right)^{-1}\right) \cdot \theta\right) d V \\
& -\sum_{i=0, \cdots, 3} \int_{\omega_{i}}\left(D_{k} \frac{\partial u_{k}}{\partial n}\right)\left(D_{k} \frac{\partial p_{k}}{\partial n}\right)^{\left(D_{k}^{i}\right)^{-2}\left(\nabla D_{k} \cdot \theta\right) d V}
\end{aligned}
$$

which reduces to

$$
s_{k, n}^{\varepsilon} \rightarrow \int_{\Gamma}\left(D_{k} \frac{\partial u_{k}}{\partial n}\right)\left(D_{k} \frac{\partial p_{k}}{\partial n}\right)\left[D_{k}^{-1}\right](\theta \cdot n) r d s .
$$

Using (27), 28) and (26), we can pass to the limit in the expression of $\partial J / \partial \omega\left(\varphi^{\varepsilon}, e\right)$ and we finally get 21].

\subsection{Gradient method}

We move the shape at each step of our algorithm with a velocity field $\theta_{n}$ belonging to a sub-space $W$ of $H^{1}\left(\Omega, \mathbb{R}^{2}\right)$ accounting for some boundary conditions. This descent direction is chosen as the unique element $\theta_{n} \in W$ such that for every $\theta \in W$,

$$
\left(\theta_{n}, \theta\right)_{W}+\left\langle\frac{\partial J}{\partial \omega}\left(\omega^{n}, e\right), \theta\right\rangle=0
$$

This step has also the property to regularize the calculated velocity field. We remark that this field $\theta_{n}$ also satisfies $\left\langle\partial J / \partial \omega\left(\omega^{n}, e\right), \theta_{n}\right\rangle=-\left\|\theta_{n}\right\|_{W}^{2}$ so that moving the sub-domains $\omega_{i}^{n}$ along this direction for a sufficiently small time step $d t$ will make decrease the value of $J(\omega, e)$.

\subsection{Volume constraint on $\omega_{0}$}

As for many structural optimization problem, it is necessary in our problem to add a volume constraint due to the level of power in the core that we want to keep constant during the optimization process. It concerns the volume of the subset $\omega_{0} \subset \omega$ consisting of fissile medium. This constraint can be imposed by the use of a Lagrange multiplier $l_{k}$. We now consider the Lagrangian associated to the volume constraint : $J(\omega, e)+l_{k}\left(V\left(\omega_{0}\right)-V_{0}\right)$ and we look for a couple $\left(\omega, l_{k}\right)$ which verifies the optimality condition 1]:

$$
\left\langle\frac{\partial J}{\partial \omega}, \theta\right\rangle+l_{k} \int_{\partial \omega_{0}}(\theta \cdot n) r d s=0 \quad \forall \theta \in W
$$

The new descent direction is computed from the following equation :

$$
\text { Find } \theta_{n} \in W \text { s.t. }\left(\theta_{n}, \theta\right)_{W}+\left\langle\frac{\partial J}{\partial \omega}\left(\omega^{n}, e\right), \theta\right\rangle+l_{k} \int_{\partial \omega_{0}^{n}}(\theta \cdot n) r d s=0 \quad \forall \theta \in W
$$

To this end, we divide $\theta_{n}$ into two components $\bar{\theta}_{n}$ and $\theta_{n}^{*}$ solutions of:

$$
\left\{\begin{array}{l}
\left(\bar{\theta}_{n}, \theta\right)_{W}+\left\langle\frac{\partial J}{\partial \omega}, \theta\right\rangle=0 \quad \forall \theta \in W \\
\left(\theta_{n}^{*}, \theta\right)_{W}+\int_{\partial \omega_{0}^{n}}(\theta \cdot n) r d s=0 \quad \forall \theta \in W
\end{array}\right.
$$


By linearity, the resulting velocity $\bar{\theta}_{n}+l_{k} \theta_{n}^{*}=\theta_{n}$ is a solution of the equation 31. From the numerical point of view, our algorithm consists in advecting the sub-domains $\omega_{i}^{n}$ along $\theta_{n}$ and checking if the volume of the next domain $\omega_{0}^{n+1}=X_{\theta_{n}}\left(\omega_{0}^{n}, \delta t\right)$ satisfies approximately the constraint, i.e $\left|\frac{\operatorname{Vol}\left(\omega_{0}^{n+1}\right)-V_{0}}{V_{0}}\right| \leq \epsilon$. If that is not the case, we adjust the multiplier $l_{k}$. More precisely, at each step of this loop, we update the value of the Lagrange multiplier by :

$$
l_{k+1}=l_{k}+\alpha \frac{\operatorname{Vol}\left(\omega_{0}^{n+1}\right)-V_{0}}{V_{0}}, \quad \alpha \in \mathbb{R}^{+} .
$$

\section{EXtension of THE MODEL TO THE 2-GROUPS ENERGY EQUATION}

We explain in this section the modifications that arise in our model when considering a different diffusion model for the neutrons, the so-called two groups energy diffusion equation. This model amounts to divide the energy spectrum into two intervals and to average the different physical quantities consequently. The main difference with the previous model is that the diffusion operator is no longer self-adjoint. The superscript 1 denotes the group of high energy while the index 2 denotes the group of lower energies.

$$
\left\{\begin{array}{lr}
-r^{-1} \operatorname{div}\left(r D^{(1)} \nabla u^{(1)}\right)+\left(\alpha^{(1)}+\beta_{r}\right) u^{(1)}=\lambda\left(\gamma^{(1)} u^{(1)}+\gamma^{(2)} u^{(2)}\right) & \text { in } \cup_{i} \omega_{i} \\
-r^{-1} \operatorname{div}\left(r D^{(2)} \nabla u^{(2)}\right)+\alpha^{(2)} u^{(2)}=\beta_{r} u^{(1)} & \text { in } \omega \\
{\left[u^{(k)}\right]=0} & \text { on } \Gamma \text { for } k=1,2 \\
{\left[\frac{\partial u^{(k)}}{\partial n}\right]=0} & \text { on } \Gamma \text { for } k=1,2 \\
u^{(1)}=u^{(2)}=0 & \text { on } \Gamma_{D}
\end{array}\right.
$$

As in the one group model, all the parameters are positive valued functions of the sodium and plutonium concentration and of the region type. This generalized eigenvalue problem satisfies a Krein and Rutman theorem [3] what proves the existence of a positive simple eigenvalue $\lambda$ with a minimal modulus and associated to an eigenvector keeping the same sign over $\Omega$. As in the 1-group case, we consider two different states corresponding to two different concentrations of sodium $d_{k}^{N a}(k=1,2)$. We adopt the following notations for denoting the stiffness and mass operator, that depend on the concentration $d_{k}^{N a}$,

$$
\begin{aligned}
& \mathbf{a}_{k}:(\mathbf{u}, \mathbf{v}) \in X^{4} \longmapsto \int_{\Omega}\left(\boldsymbol{D}_{k} \nabla \mathbf{u} \cdot \nabla \mathbf{v}+\boldsymbol{\alpha}_{k} \mathbf{u} \cdot \mathbf{v}\right) d V \in \mathbb{R} \\
& \mathbf{b}_{k}:(\mathbf{u}, \mathbf{v}) \in X^{4} \longmapsto \int_{\Omega} \gamma_{k} \mathbf{u} \cdot \mathbf{v} d V \in \mathbb{R},
\end{aligned}
$$

where $\mathbf{u}=\left(u^{(1)}, u^{(2)}\right)^{T} \in X^{2}, \mathbf{v}=\left(v^{(1)}, v^{(2)}\right)^{T}$, for $k=1,2$

$$
\boldsymbol{D}_{k}=\left(\begin{array}{cc}
D_{k}^{(1)} & 0 \\
0 & D_{k}^{(2)}
\end{array}\right) ; \quad \boldsymbol{\alpha}_{k}=\left(\begin{array}{cc}
\alpha_{k}^{(1)}+\beta_{r, k} & 0 \\
-\beta_{r, k} & \alpha_{k}^{(2)}
\end{array}\right) ; \quad \gamma_{k}=\left(\begin{array}{cc}
\gamma_{k}^{(1)} & \gamma_{k}^{(2)} \\
0 & 0
\end{array}\right)
$$

The weak form of the 2 groups energy equation is given in the following proposition.

Proposition 3.1. The weak form of equation (34) is :

$$
\text { Find }\left(\mathbf{u}_{k}, \boldsymbol{\lambda}_{k}\right) \in V^{2} \times \mathbb{R} \text { such that } \mathbf{a}_{k}\left(\mathbf{u}_{k}, \mathbf{v}_{k}\right)=\boldsymbol{\lambda}_{k} \mathbf{b}_{k}\left(\mathbf{u}_{k}, \mathbf{v}_{k}\right) \forall \mathbf{v}_{k} \in V^{2}
$$


The rate of fission can be evaluated thanks to the solution $\mathbf{u}$ by considering the quantity $\boldsymbol{\tau}_{1}=\boldsymbol{\beta}_{1}^{0} \cdot \mathbf{u}_{1}$, where $\boldsymbol{\beta}:=\nu^{-1} \boldsymbol{\gamma}$. In this case, we use the same form for the cost functions. More precisely, we set

$\boldsymbol{J}_{1}(\varphi, e):=\boldsymbol{\lambda}_{1}(\varphi, e)-\boldsymbol{\lambda}_{2}(\varphi, e), \quad \boldsymbol{J}_{2}(\varphi, e):=\left(\boldsymbol{\lambda}_{1}(\varphi, e)-\lambda_{0}\right)^{2} . \quad \boldsymbol{J}_{3}(\varphi, e):=\operatorname{Vol}\left(\varphi_{0}\right)^{\frac{s-1}{s}}\left(\int_{\Omega} \boldsymbol{\tau}_{1} d V_{0}\right)^{-1}\left|\int_{\Omega} \boldsymbol{\tau}_{1}^{s} d V_{0}\right|^{1 / s}$,

and consider the minimization problem

$$
\underset{(\omega, e) \in \mathcal{U}_{a d}}{\arg \min } \boldsymbol{J}:=s_{1} \boldsymbol{J}_{1}+\boldsymbol{J}_{2}+s_{3} \boldsymbol{J}_{3}
$$

Obviously, the minimization problem obtained in this 2-group case is formally equivalent to the more simple 1-group case. Thus, the expression of the derivative of $J$ with respect to $e$ or $\omega$ does still apply in this case, up to replace all variables by their vectorial counterpart (that is replacing $u$ by $\mathbf{u}, D$ by $\boldsymbol{D}$ and so on).

\section{Numerical RESUlts}

The coefficients $D, \alpha$ and $\beta$ will be taken constant in each region $\omega_{i}$ with $i=1, \cdots 3$ and will vary in $\omega_{0}$ in a bounded interval, the percentage of Plutonium $e$ being chosen in the interval $[15 \%, 27 \%]$.

In the table (1) are given the dependency of each coefficient with respect to $e$.

TABLE 1. Interpolations of some ERANOS values for the coefficients in the set $[15 \%, 27 \%]$

\begin{tabular}{|c|c|c|c|}
\hline Coefficient & & Nominal state & Perturbed state \\
\hline$D$ & hyperbolic & $\frac{1}{-5,029649.10^{-3} e+7,898226.10^{-1}}$ & $\frac{1}{-5,025644.10^{-3} e+7,889087.10^{-1}}$ \\
\hline$\alpha$ & linear & $7,819137.10^{-5} e+4,750344.10^{-3}$ & $7,828342.10^{-5} e+4,745445.10^{-3}$ \\
\hline$\beta$ & linear & $1,342218.10^{-4} e+4,028949.10^{-4}$ & $1,342570.10^{-4} e+4,018440.10^{-4}$ \\
\hline$\gamma=\nu \beta$ & linear & $4,066337.10^{-4} e+9,710325.10^{-4}$ & $4,067438.10^{-4} e+9,679273.10^{-4}$ \\
\hline
\end{tabular}

The optimization method for the one-group energy equation described in section 2 is here carried out with the help of the software FreeFem++. The equations of the continuous model are discretized with the finite element method on an initial mesh of $3.10^{3}$ nodes. We have considered a domain $\omega$ whose dimensions are given in the table $(2)$.

The state $u$ and the adjoint state $p$ are discretized with $P 1$ continuous finite elements. The coefficients $(D, \alpha, \gamma)$ are discretized with $P 1$ discontinuous finite elements (see $[7]$ ). The velocity field $\theta$ is discretized with $P 1 \times P 1$ finite elements.

TABle 2. Dimensions of $\omega$

\begin{tabular}{|c|c|c|c|c|}
\hline material & $\begin{array}{c}\text { internal } \\
\text { radius } \\
(\mathrm{cm})\end{array}$ & $\begin{array}{c}\text { external } \\
\text { radius } \\
(\mathrm{cm})\end{array}$ & $\begin{array}{c}\text { inferior } \\
\text { height } \\
(\mathrm{cm})\end{array}$ & $\begin{array}{c}\text { superior } \\
\text { height } \\
(\mathrm{cm})\end{array}$ \\
\hline Fissile & 0 & 150 & 60 & 140 \\
\hline Fertile & 0 & 150 & 30 & 60 \\
\hline Plénum sodium & 0 & 150 & 140 & 170 \\
\hline Reflector & \multicolumn{2}{|c|}{ thickness of $30 \mathrm{~cm}$ around the active core } \\
\hline
\end{tabular}

Between each iteration of the geometric gradient step, we perform two iterations of the parametric optimization. The different criteria are weighted with the following factors : $s_{1}=10^{5}, s_{2}=200$ and $s_{3}=8$. The target 



Figure 1. Initial design versus the optimal one at $N_{\text {iter }}=3000$ with $\delta \rho=-29 \mathrm{pcm}$ and $\langle e\rangle=17,2 \%$. On the left hand side, the surrounding subdomain is occupied by the reflector (displayed in blue), the core is made of three parts: Fertile area (bottom, red), Fissile (center, green) and Plénum (top, black). The geometry, together with the concentration of plutonium (high concentration in purple, low in orange) of the optimized designed is represented on the right hand side. Note that the topology is preserved during the optimization process.

value for the minimal eigenvalue $\lambda_{0}$ is set at 0.9 and the volume constraint must be satisfied with a $10 \%$ margin of error. The Lagrange multiplier for the volume constraint is initialized at the value $l_{0}=1$.

Note that we obtain very smooth interfaces due to a high value of the regularization factor $\xi=10^{4}$ (the constant value in front of the Laplacian term involved in the calculation of the descent direction $\theta$ ) where we consider the following scalar product on $W$ :

$$
\left(\theta_{1}, \theta_{2}\right)_{W}=\int_{\omega_{1}}\left(\xi \nabla \theta_{1}: \nabla \theta_{2}+\theta_{1} \cdot \theta_{2}\right) d V
$$

The step descent are chosen as follows :

$$
\mu=10^{4}, d t=10^{2}
$$

Applying our method during 3000 iterations leads to the design presented on figure (1) The sodium void effect $\delta \rho:=\rho_{2}-\rho_{1}$ is expressed in terms of $p \mathrm{~cm}$ which is a unit defined as $1 \mathrm{pcm}=10^{-5}$. The accuracy of the calculation for $\delta \rho$ is about $1 \mathrm{pcm}$. Note that the sodium void effect decreases from an initial value of $11 \mathrm{pcm}$ (evaluated with an uniform value of $e$ set at $20 \%$ ) to a final value of $-29 \mathrm{pcm}$. During this simulation, one may check the convergence by paying attention to the behavior of the following quantities

- for the shape, $\frac{J^{\prime}\left(\omega_{n}\right)\left(\theta_{n}\right)}{J^{\prime}\left(\omega_{0}\right)\left(\theta_{0}\right)}$

- for the content of Plutonium, $\delta e^{n}=\int_{\omega_{0}}\left(e^{n}(r, z)-e^{n-1}(r, z)\right)^{2} d V$.

The first of these quantities is represented on the figure (3) and, as expected, it strongly decreases at the beginning of the algorithm. We observe a good convergence for the Plutonium percentage $e$ as it may be seen on the figure (5). It appears that our model cannot optimize the shape factor (red curve on figure (2)) as well as the sodium void effect (represented with the blue curve). Nevertheless, this last term reach very low negatives values which demonstrates the efficiency of the geometric shape optimization method for our problem. One may verify the physical consistency of this simulation by plotting the neutrons leakages through the external 


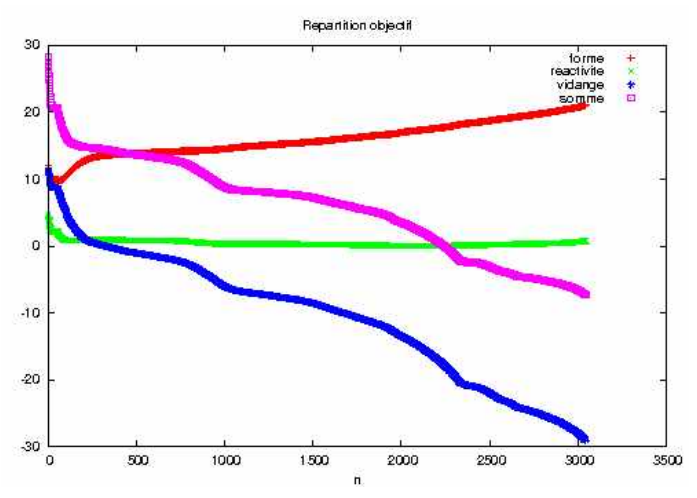

FIgURE 2. $J=\alpha_{i} J_{i}$



Figure $4 . \quad-\int_{\omega_{0}} r \operatorname{div}\left(D_{2} \nabla \phi_{2}\right) d r d z$

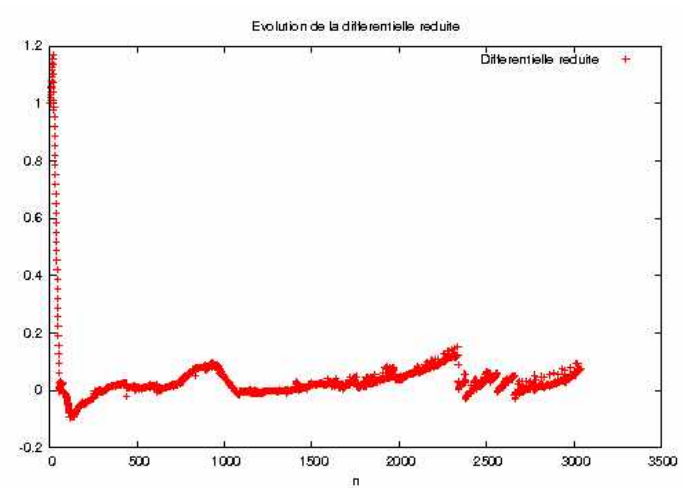

FiguRE $3 . \quad J^{\prime}\left(\omega_{n}\right)\left(\theta_{n}\right)$

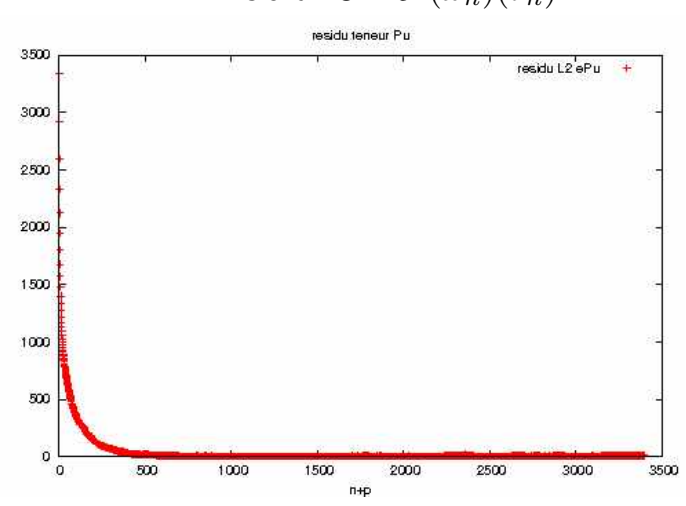

Figure 5. $L^{2}$ residual of $e^{n}(x)$

surface of the fissile region $\partial \omega_{0}$ in perturbed conditions. The figure (4) stands in good agreement with the physical explanation of the sodium void effect $\delta \rho$ : we minimize the sodium void effect by increasing its leakage component.

Another drawback of the geometric shape optimization method is that it does not account for changes of topology of the mesh what cause sometimes the superposition of the boundaries. To get rid of those overlapping boundaries, we introduce a function whose zero Level-Set match the interface. After a regularization of this function, we determine a parametrization of the regularized zero Level-Set and remesh the domain $\Omega$ accordingly. Our algorithm is then restarted with the modified topology. Note that we are here talking of the topology of the mesh. For instance, the mesh displayed on the right hand side of Figure 6 has a different topology than the one of the initial mesh - better seen on Figure 1 - because the interfaces do connect different parts of the surrounding box. This regularization step is represented on the figure (6). We re-initialize the percentage of Plutonium at the mean value reached at the end of the first optimization process and perform again our method. It leads to the design of figure (7).

We reach this time a sodium void effect $\delta \rho=-35 \mathrm{pcm}$ but we observe that the shape did not have changed that much. Due to a good behavior of the convergence indicators (figures (9), (10)), we think that we probably have reached a local minimizer for our optimization problem.

\section{CONCLUSION}

This works gives an overview of the application of a geometric shape optimization to the design of a sodium fast reactor. Although some work still needs to be done in order to improve the multi-criteria convergence, we 

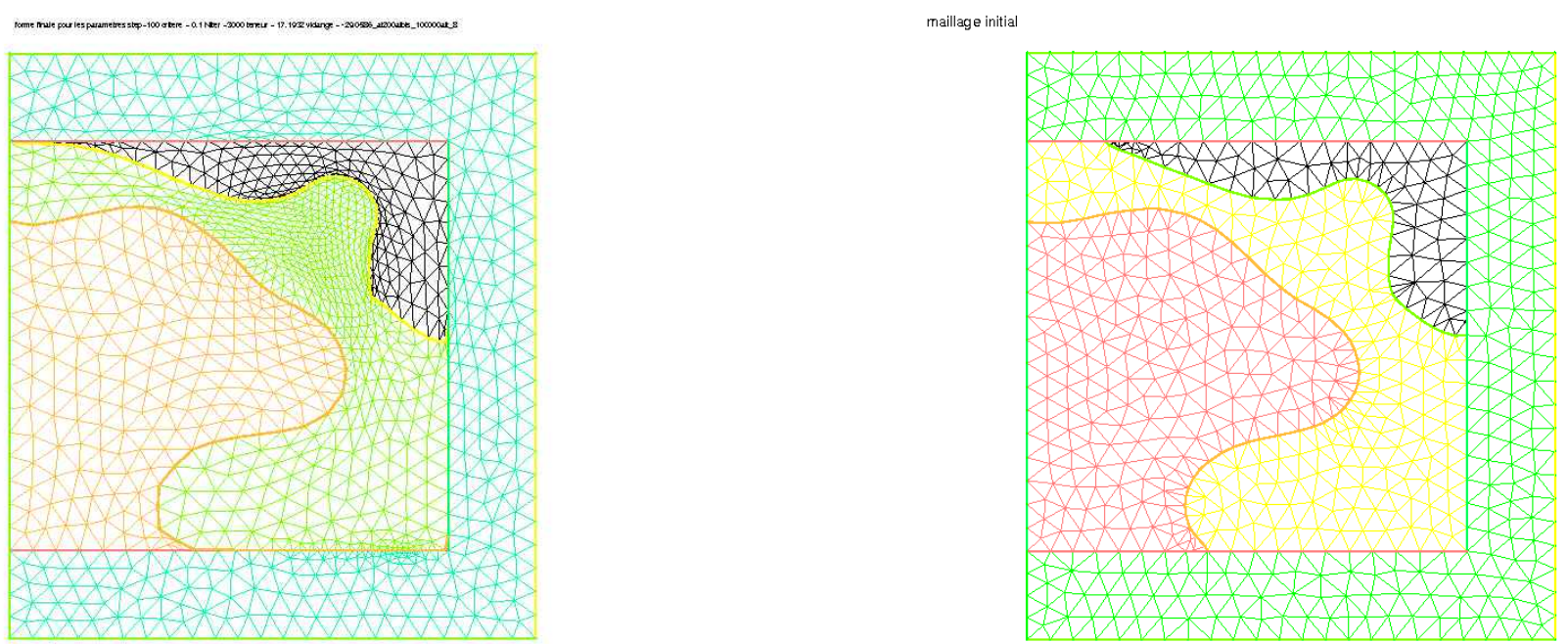

FiguRE 6. Boundary superposition (on the left) is removed using a regularization by the mean of the introduction of a Level-Set function.

observe that our algorithm leads to a very efficient optimization of the sodium void effect and a really strong change of the shape. In addition, we should also study the influence of the initial guess on our result which is an important point in geometric optimization. The use of the two groups energy equation needs to be tested in the future in order to enhance the accuracy of the state equation. Another step toward more relevant results from an industrial point of view would be to add in the algorithm some geometrical constraints. Those constraints would be chosen in order to take into account the size and the shape of the nuclear assemblies. This can be done either by adding some constraints directly in the algorithm or by performing a penalization method on the final shape (see [2]) .

This work was partially support by the PEPS-CNRS project OPTFOR.

\section{REFERENCES}

[1] G. Allaire. Conception optimale de structures, volume 58. Springer Verlag, 2007.

[2] G. Allaire and C. Castro. A new approach for the optimal distribution of assemblies in a nuclear reactor. Numerische Mathematik, 89(1):1-29, 2001.

[3] G. Allaire and C. Castro. Optimization of nuclear fuel reloading by the homogenization method. Structural and Multidisciplinary Optimization, 24(1):11-22, 2002.

[4] G. Allaire, F. Jouve, and A.M. Toader. Structural optimization using sensitivity analysis and a level-set method. Journal of computational physics, 194(1):363-393, 2004.

[5] O. Pantz. Sensibilité de l'équation de la chaleur aux sauts de conductivité. Comptes Rendus Mathematique, 341(5):333-337, 2005.

[6] O. Pironneau. Optimal shape design for elliptic systems. System Modeling and Optimization, pages 42-66, 1982.

[7] O. Pironneau, F. Hecht, A. Le Hyaric, and J. Morice. Freefem++. See http://www. freefem. org/ff+, 2009.

[8] P. Reuss. Précis de neutronique. L'Editeur: EDP Sciences, 2003. 


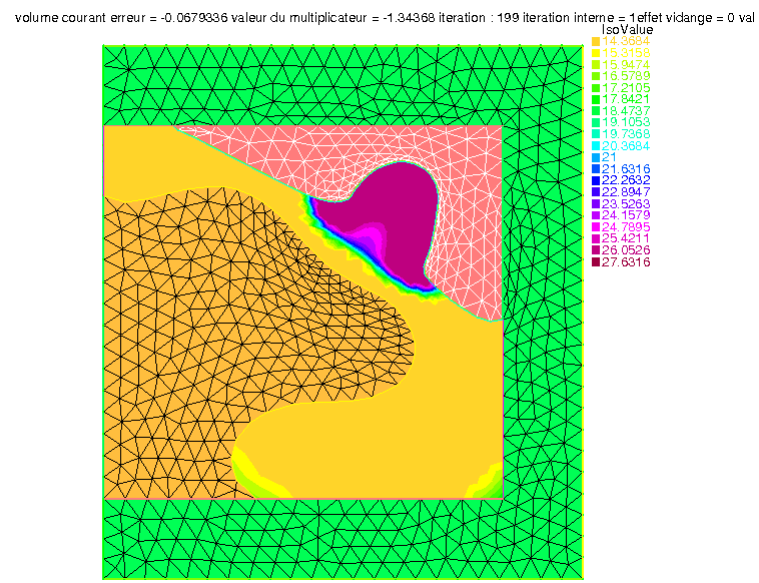

Figure 7. New design of the core



FiguRE 9. $\frac{J^{\prime}\left(\omega_{n}\right)\left(\theta_{n}\right)}{J^{\prime}\left(\omega_{0}\right)\left(\theta_{0}\right)}$

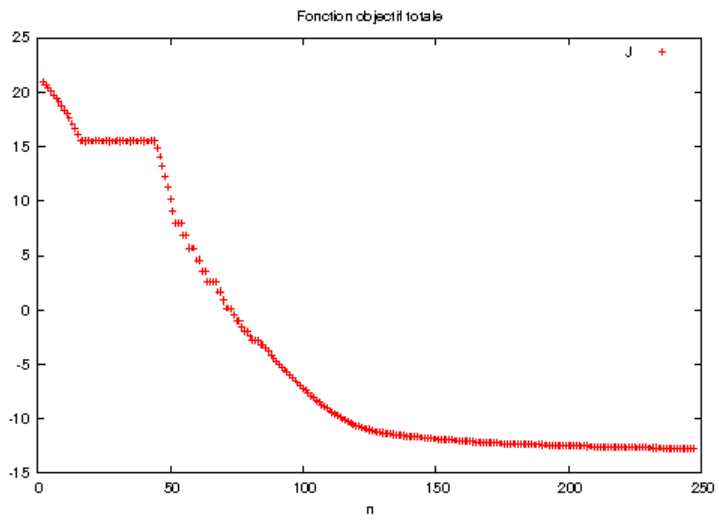

FIgURE 8. History of $J$



Figure 10. $L^{2}$ residual of $e^{n}(x)$ 